The Journal of Rheumatology 2021;xx:xxxx

doi:10.3899/jrheum.210992

First Release October 152021

\section{Dr. Kremer et al reply}

\section{To the Editor:}

Drs. Pincus, Bergman, and Yazici have raised some concerns about our published article comparing the Clinical Disease Activity Index (CDAI) with simultaneous measures of the Routine Assessment of Patient Index Data 3 (RAPID3). ${ }^{1}$

We believe our publication has clearly established that the validated CDAI scores provide a fundamentally different evaluation of disease status compared with the RAPID3. The differences in the final metrics from these different scoring systems, when compared in a very large number of patients, are quite meaningful. ${ }^{1}$ The implications of these differences, if used to inform decisions about treating to target, ${ }^{2}$ are important. It is also academically relevant to note the number of patients we studied from the Corrona and Brigham and Women's Rheumatoid Arthritis Sequential Study (BRASS) registries was 49,598 from a combination of over 700 rheumatologists at 184 sites over a 20-year interval. ${ }^{1}$ The publication Pincus et al use to compare data in their letter are derived from a combined total of 285 patients from the practices of each of the authors. ${ }^{2}$ It is unlikely that 285 patients can represent the entire rheumatoid arthritis (RA) population of 3 practices. It is thus appropriate to reexamine the validity of the RAPID3 from a much larger, and more diverse, population.

Consider these fundamentals: The treatment of RA to target is universally accepted. ${ }^{3}$ If we are to treat to target, then we should have a consensus about how to define the target. The clinical manifestations of RA-associated inflammation are tender and swollen joints on examination. The treating rheumatologist's judgment of global disease activity, not found in the RAPID3, is equally weighted with the physical examination in the CDAI. A rheumatologist treats hundreds, perhaps thousands, of patients with RA over many years. This experience informs an expert professional judgment on what constitutes disease severity. It would thus logically follow that the medical specialist is better positioned to judge the relative activity of the disease they are treating than a single patient without any frame of reference other than a personal perception of their relative welfare.

The published literature describes many challenging issues associated with the patient global assessment (PtGA) of disease. ${ }^{1,4,5,6,7,8}$ This literature is derived from diverse geographic, cultural, and ethnic sources. These publications demonstrate that noninflammatory human emotions such as anxiety, depression, and lack of life satisfaction drive the PtGA. The published literature is coherent, consistent, and compelling. ${ }^{1,4,5,6,7,8}$ Thus, the complete reliance on a patient-only metric, without input from either a physician global assessment (PGA) or examination of tender and swollen joints, will inevitably skew evaluations of inflammatory disease status. We examined correlations of individual components of both the CDAI and the RAPID3 with the complete metric score derived from either of these systems. For the PGA, $r=0.78$ with the CDAI and $r=0.47$ for the Corrona RAPID3. ${ }^{1}$

There is a consensus in the published literature that RA is an inflammatory autoimmune disease and not a noninflammatory, psychological construct. It must also be noted in this same context that all international regulatory agencies require physician-derived evaluations of tender and swollen joint counts (integrated into American College of Rheumatology criteria and Disease Activity Score in 28 joints) for approval of new drugs. Of additional relevance, the worldwide literature indicates that noninflammatory psychological measures related to depression, anxiety, and absence of life satisfaction are present in chronic diseases that do not share a common pathophysiology. ${ }^{9}$

The low $\kappa$ levels of agreement of RAPID3 with CDAI, together with the very disparate correlations of the PtGA and PGA with the full scores from each metric, do not support the use of a patient-only system such as the RAPID3 for making treatment decisions. Should the patients themselves provide all that is required to make treatment decisions without any input from the treating physician who employs the same physical examination metrics approved by all regulatory agencies?

The very same RAPID3 used in RA has been purported to be useful in evaluating patients with osteoarthritis (OA). ${ }^{10}$ How can the identical metric provide an accurate assessment of disease activity in these very different disease states? The answer is simple: Both RA and OA are chronic diseases associated with depression and low patient life satisfaction. Of course, many patients with RA may also have OA. If the RAPID3 can be used as a reliable metric in patients with both conditions, then which disease is driving the resultant score?

We also suspect that the focus on patient pain and global activity would result in scores for patients with fibromyalgia (FM) that would mimic those from patients with RA. Many patients with RA have elements of FM.

We should all aspire to improve our approach to noninflammatory components of the patients we treat. Clinicians may consider the RAPID3 as a potentially useful supplementary tool to provide some sense of the noninflammatory, psychological dimensions of the complex human beings we treat.

Joel M. Kremer ${ }^{1}$ (D), MD

George Reed ${ }^{2}$ (D) $\mathrm{PhD}$

Dimitrios A. Pappas ${ }^{3}$ (D) MD, MPH

Kevin Kane ${ }^{2}$ (D), MS

Vivi L. Feathers ${ }^{4}$ (i), MS

Michael E. Weinblatt ${ }^{4}$ (D), MD

Nancy Shadick ${ }^{4}$ (D), MD, MPH

Jeffrey Greenberg ${ }^{5}$ (D) $\mathrm{MD}, \mathrm{MPH}$

Leslie L. Harrold ${ }^{2}$ (D), MD, MPH

${ }^{1}$ Albany Medical College, Albany, New York;

${ }^{2}$ University of Massachusetts School of Medicine, Worcester, Massachusetts;

${ }^{3}$ Columbia University College of Physicians and Surgeons, New York, New York;

${ }^{4}$ Brigham and Women's Hospital, Boston, Massachusetts; ${ }^{5}$ New York University School of Medicine, New York, New York, USA. 
This study was supported by the Corrona Research Foundation, a not-for-profit 501(c)3 United States foundation.

The authors declare no conflicts of interest relevant to this article.

Address correspondence to Dr. J.M. Kremer, MD, 16 Hillard Lane,

Latham, NY 12110, USA. Email: jkremer@corrona.org.

\section{REFERENCES}

1. Kremer JM, Pappas DA, Kane K, et al. The Clinical Disease Activity Index (CDAI) and the Routine Assessment of Patient Index Data 3 (RAPID3) for achievement of treatment strategies in rheumatoid arthritis. J Rheumatol xxxxxxxxx.

2. Pincus T, Bergman MJ, Yazici Y. Should quantitative measures and management of rheumatoid arthritis include more than control of inflammatory activity? J Rheumatol xxxxxxxxx.

3. Fraenkel L, Bathon JM, England BR, et al. 2021 American College of Rheumatology guideline for the treatment of rheumatoid arthritis. Arthritis Care Res 2021;73:924-39.

4. Ferreira RJ, Duarte C, Ndosi M, de Wit M, Gossec L, da Silva JA. Suppressing inflammation in rheumatoid arthritis: does patient global assessment blur the target? A practice-based call for a paradigm change. Arthritis Care Res 2018;70:369-78.
5. Studenic P, Radner H, Smolen JS, Aletaha D. Discrepancies between patients and physicians in their perceptions of rheumatoid arthritis disease activity. Arthritis Rheum 2012;64;2814-23.

6. Hirsh J, Wood P, Keniston A, et al. Limited health literacy and patient confusion about rheumatoid arthritis patient global assessments and model disease states. Arthritis Care Res 2019;71:611-9.

7. Marrie RA, Hitchon CA, Walld R, et al; Canadian Institutes of Health Research Team in Defining the Burden and Managing the Effects of Psychiatric Comorbidity in Chronic Immunoinflammatory Disease. Increased burden of psychiatric disorders in rheumatoid arthritis. Arthritis Care Res 2018;70:970-8.

8. Barton JL, Imboden J, Graf J, Glidden D, Yelin EH, Schillinger D. Patient-physician discordance in assessments of global disease severity in rheumatoid arthritis. Arthritis Care Res 2010;62:857-64.

9. Cassileth BR, Lusk EJ, Strouse TB, et al. Psychosocial status in chronic illness-a comparative analysis of six diagnostic groups. N Engl J Med 1984;311:506-11.

10. Chua, JR, Jamal S, Riad M, et al. Disease burden in osteoarthritis is similar to that of rheumatoid arthritis at initial rheumatology visit and significantly greater six months later. Arthritis Rheumatol 2019;71:1276-84. 